CARNETS DE Carnets de géographes

GÉOGRAPHES.

11 | 2018

Varia

\title{
La friche militaire urbaine, un nouvel espace convoité ?
}

Stéphanie Lotz-Coll

\section{OpenEdition}

Journals

Édition électronique

URL : https://journals.openedition.org/cdg/1443

DOI : $10.4000 /$ cdg. 1443

ISSN : 2107-7266

Éditeur

UMR 245 - CESSMA

Référence électronique

Stéphanie Lotz-Coll, « La friche militaire urbaine, un nouvel espace convoité ? 》, Carnets de géographes [En ligne], 11 | 2018, mis en ligne le 15 septembre 2018, consulté le 20 mai 2021. URL : http:// journals.openedition.org/cdg/1443; DOI : https://doi.org/10.4000/cdg.1443

Ce document a été généré automatiquement le 20 mai 2021.

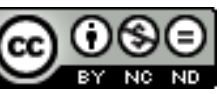

La revue Carnets de géographes est mise à disposition selon les termes de la Licence Creative Commons Attribution - Pas d'Utilisation Commerciale - Pas de Modification 4.0 International. 


\title{
La friche militaire urbaine, un nouvel espace convoité ?
}

\author{
Stéphanie Lotz-Coll
}

1 La ville est un phénomène ancien résultant de transformations successives au cours de l'histoire (Bairoch, 1985) et les affirmations se recoupent fréquemment pour définir les principes des évolutions urbaines les plus récentes. Ainsi, Fernandez et Cornes (1990) en montrant les modalités de construction et les évolutions urbaines d'une ville imaginaire considèrent que l'extension urbaine résulte de la réutilisation successive de quartiers ou de terrains ayant déjà connu un premier usage et ont ainsi permis à la ville d'évoluer. Leonardo Benevolo (1993) affirme, quant à lui, que "l'histoire des villes européennes et l'histoire de l'Europe sont, dans une large mesure, une seule et même aventure [...] », celle des mutations d'un système productif qui, en impactant la société, ont pour conséquences de profonds changements urbanistiques et un bouleversement de la hiérarchie des villes.

2 Les villes sont le fruit de l'activité des hommes : elles reflètent donc les évolutions de la société et s'adaptent de façon cyclique aux mutations des modes de production ou de l'habitat. La notion de « recyclage » des terrains ou bâtiments urbains devenus inutiles est importante dans l'histoire des villes.

Cependant, si la réutilisation des espaces en cessation d'activité peut aujourd'hui sembler évidente, elle n'a pas toujours été envisagée ainsi et la perception des friches a évolué dans le temps. Sur le plan opérationnel, la réintégration de la friche dans la dynamique urbaine, bien que considérée comme nécessaire, n'en reste pas moins complexe. L'augmentation du nombre de friches a également mis en évidence les problèmes non résolus qu'elles impliquent tels la pollution liée à leur activité initiale, le besoin de sécuriser un site non gardienné, la nécessaire mise en conformité avec les normes existantes ou encore les possibilités d'occupation illicites offertes par ces sites désaffectés.

Dans ce contexte, les anciens sites militaires font figures d'exception car leur ancienne activité pousse à considérer la friche militaire urbaine avec une défiance souvent liée à la méconnaissance du site totalement clôturé du temps de son activité militaire. Dans le 
cadre de cet article, il s'agira d'envisager, si la friche militaire urbaine peut représenter une opportunité foncière pour repenser le territoire urbain alors que, dans une démarche de concurrence interurbaine, la demande et l'offre d'espaces s'inversent fréquemment: autrefois, un besoin était satisfait par un lieu pour y répondre, aujourd'hui, un espace est disponible et il faut lui trouver une nouvelle vocation en accord avec les besoins urbains.

5 Pour répondre à cette question, nous étudierons la friche comme objet urbain passant d'une logique de répulsion à celle d'opportunité, puis nous envisagerons dans le détail la friche militaire et la façon dont sa reconversion peut bénéficier à une ville qui subit les conséquences du départ des militaires.

\section{La friche, un objet d'intérêt pour les géographes et les urbanistes}

6 Le mot friche désigne initialement une "terre non cultivée ", terme qui relève du vocabulaire agricole dans le langage courant. Il conserve une connotation négative, étant associé à des périodes au cours desquelles l'agriculteur n'avait pas l'utilité de son champ ou laissait la terre se reposer. Aujourd'hui, ce mot, s'il conserve son sens premier, s'inscrit dans une acception plus large :» surface laissée à l'abandon, non encore construite ou supportant des constructions insalubres, en milieu urbain » (Saffache, 2005). Il intègre ainsi des espaces marqués par l'homme. L'évolution sémantique a donc permis le développement d'un sens figuré lié à l'abandon d'une activité puis, par logique d'assimilation, de l'expression «friches industrielles » (ibid.). Depuis la fin du XXème siècle, le vocabulaire des géographes s'est également enrichi de l'expression de «friches urbaines " désignant les «terrains laissés à l'abandon en milieu urbain, en attendant un réemploi hypothétique » (Dorier-Apprill, $2001: 36$ ).

7 De nombreux facteurs influencent cette friche urbaine catégorisée en fonction de différents critères (Bianchetti, 1985) tels son statut d'abandon, son niveau de dégradation ou encore son temps d'inutilité. À ces trois critères principaux s'ajoutent la superficie de la friche ou les possibilités d'affectation transitoire dans le cadre du processus de réhabilitation.

8 Cette classification implique une grande variété de situations. Outre ces éléments, il est commun de qualifier les friches en fonction de leur origine.

\section{Les différents types de friches}

9 Bien que chaque friche soi tunique du fait de sa situation, de son histoire et des enjeux qu'elle représente, une typologie de principe peut être établie (Communautés urbaines de France, 2011) en insistant sur des caractéristiques permettant une mise en perspective de la friche militaire :

10 Les friches industrielles constituent un ensemble varié. Leur structure bâtie est parfois complexe et la pollution du sol peut être très élevée.

11 Les friches ferroviaires concernent principalement les sites liés aux4000 km de voies de chemin de fer inutilisés. Ce patrimoine suscite souvent l'envie des collectivités. D'autres bâtiments techniques liés à l'activité ferroviaire complètent cet ensemble (Giraud, 2006). 
12 Les friches portuaires regroupent les quais, les chantiers navals ou les hangars des villes portuaires de l'après-guerre souvent caractérisées par une organisation sectorielle séparant les espaces urbain et portuaire de la zone industrielle associée. A partir des années 1970-1980, l'effondrement de l'industrie lourde a généré de nombreuses friches portuaires dont la reconversion a tardé à se mettre en place car leur traitement est complexe, impliquant un véritable décloisonnement de l'interface ville-port avec une réouverture du port sur la ville (Tiano, 2010).Les friches administratives et d'équipements publics regroupent un ensemble diversifié comprenant des bâtiments non utilisés par l'État ou les collectivités (Rey-Lefebvre, 2015).Les friches hospitalières résultent de stratégies de concentration ou de relocalisation, et concernent parfois de vastes espaces enclavés (Pascal et Kostrzewa, 2017). Les friches d'habitat et commerciales, fréquentes, peuvent s'étendre sur des quartiers entiers.

\section{La friche, un objet urbain complexe}

13 Au-delà de sa stricte définition, la friche, recouvre des problématiques diverses lorsqu'on s'intéresse à sa reconversion, tant en termes de temporalité urbaine qu'au niveau des défis et exigences qu'elle implique : les friches urbaines sont soumises à des processus de mutation variés caractérisés par la régularité d'apparition de trois phases (Ambrosino et Andres, 2008) et complétés parun quatrième temps peu mis en valeur jusqu'à présent (figure 1) :

14 L'avant-friche est une période transitoire avec une baisse progressive de l'activité du site dont la fonction initiale reste inchangée.

15 C'est le cadre historique de la friche. Il permet de conserver la mémoire du site et l'attachement patrimonial afférent. La friche prend alors une importance accrue pour deux acteurs centraux, la collectivité locale et le propriétaire qui définissent, conjointement ou non, des orientations initiales pour la friche. L'éventualité de sa rentabilité à la vente est abordée, la friche peut alors devenir une ressource foncière.

La veille, période la plus active et normalement la plus longue, est la phase transitoire entre l'abandon du site et son projet de réutilisation (planifié). C'est un temps de réflexion sur les possibilités d'évolution de la friche avec deux cas :

17 La friche ne fait l'objet d'aucun intérêt: seuls les processus inhérents à la friche, la reconquête végétale ou la dégradation naturelle du bâti existent. La friche se marginalise alors du tissu urbain ;

18 L'intérêt pour la friche est avéré : des acteurs habituellement absents des processus de planification urbaine tels le patronat, les grandes entreprises publiques ou les milieux artistiques, évaluent le devenir du site. Le site est alors une friche en projet.

19 Dans ce contexte, la friche est à la fois symbole de permissivité pour des milieux marginaux et ressource foncière pour la ville.

20 L'après-friche est la phase de reconversion du site : les différents acteurs réajustent les paramètres de la phase de veille et la friche devient une ressource foncière pour laville durable. Des stratégies liées à l'attractivité du site à reconvertir, développées lors de la phase de veille, sont enrichies par des éléments réactualisés, parfois dans le cadre du marketing urbain pour répondre aux enjeux de concurrence entre les villes. 
Ces trois phases existent quel que soit le statut de la friche concernée. Cependant, leur durée et la nature des acteurs impliqués peuvent varier. Ce sont donc des critères pour la réussite de la requalification d'une friche.

Dans le cadre des travaux sur l'effet levier de la reconversion des sites militaires (Lotz, 2017), un quatrième temps de la friche a dû être envisagé lorsque les projets de reconversion ont abouti : le temps de la friche reconvertie. Il permet d'évaluer les effets de sa reconversion, en termes économique, social et urbain. Le nouvel espace doit alors répondre à de nombreuses questions :

L'impact du départ de l'activité initiale a-t-il été compensé ?

L'emprise reconvertie participe-t-elle à la revitalisation/redynamisation économique et sociale du territoire?

25 L'emprise reconvertie s'intègre-t-elle dans le projet de la ville et a-t-elle acquis une nouvelle dimension urbaine de façon générale?

Ce dernier temps est souvent sous-estimé car il se confronte aux temps de la ville, et en particulier au moyen terme du mandat électif, par opposition au long terme de la politique foncière et de la planification urbaine (Ambrosino, 2008). Cependant, il importe que la réussite de la reconversion du site marque durablement le territoire pour que cet espace ne redevienne pas une friche à court terme et pour faciliter la reconversion d'autres friches en intégrant le retour d'expérience ou en appliquant un processus de planification déjà éprouvé. Le cas de friches transformées en zones commerciales est un exemple de l'enjeu de cette dernière phase : si le besoin a été surévalué, les locaux peuvent rester inoccupés avant de revenir des friches. On peut ainsi citer le cas du Parc de loisirs Cap Découverte (Haschar-Noé, 2008). Lors de la fermeture de la dernière mine de charbon du Tarn, les élus locaux ont choisi d'y maintenir une activité économique en transformant le site de la mine à ciel ouvert en une base et parc de loisirs au prix d'un investissement de $66 \mathrm{M€}$. Les prévisions initiales de fréquentation anticipaient 660000 entrées payantes et la création de 250 emplois directs et de 1000 emplois indirects. Mais la fréquentation du site (environ 20000 visiteurs annuels) ne permit jamais d'atteindre ces résultats. Face à l'ampleur des pertes financières, le site a d'abord réduit ses activités en 2013 avant de fermer. Il accueille des déchets faiblement radioactifs depuis 2016. 
Figure 1 : les temps de la friche

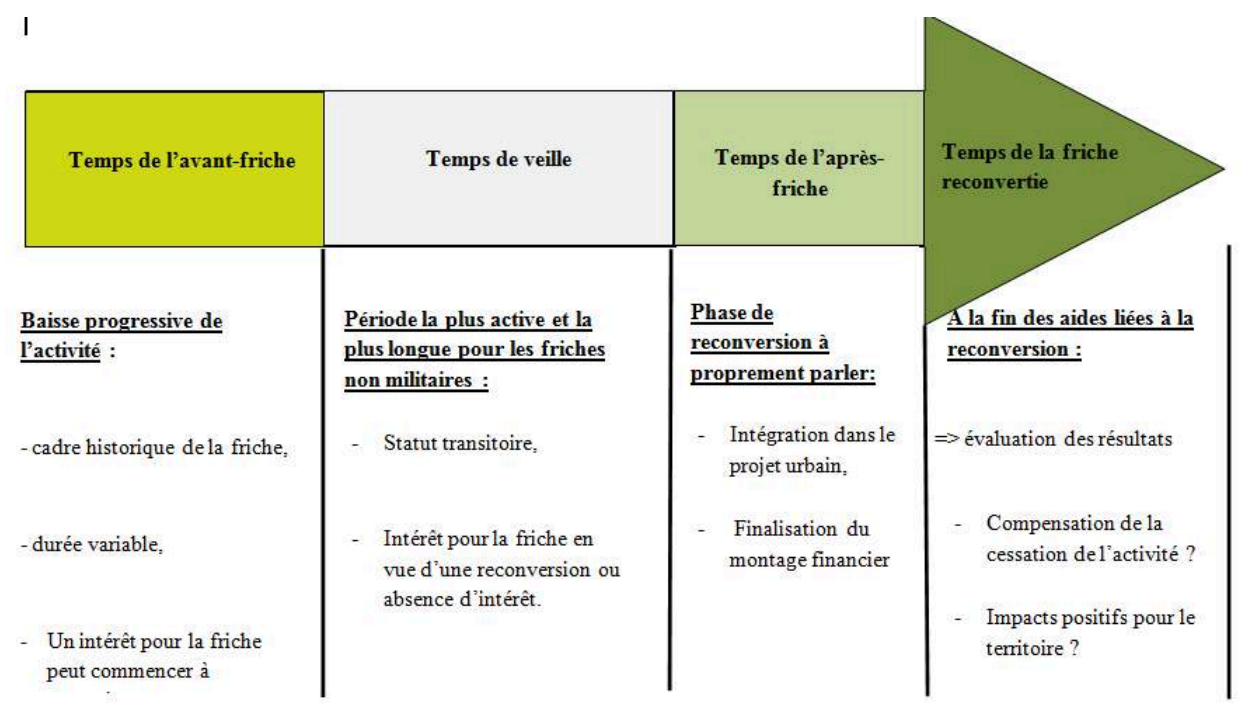

\section{La friche, une relation ambiguë à la ville}

La mutabilité de la ville lui a permis de durer dans le temps. Ainsi, de façon générale, dans les années 1920, les friches religieuses et militaires ont facilement été reconverties en école ou en mairie, les friches industrielles à partir des années 1960-70 et enfin les friches commerciales ou d'habitat à partir des années 1980, ont fréquemment permis aux villes de se renouveler et d'envisager de nouvelles activités.

Cependant, au-delà des inconvénients intrinsèques liés à sa nature, la friche a vu sa perception évoluer. D'élément indispensable au maintien des dynamiques urbaines, elle est devenue un handicap lorsque sa fréquence d'apparition s'est accélérée.

De fait, à l'issue de la période dite des Trente Glorieuses, la ville a connu ses premières transformations brutales avec une apparition massive de friches. Il s'agissait d'abord de friches industrielles dont la connotation négative était symbolique car elles représentaient le témoignage d'une crise économique et sociale, et matérialisaient les stigmates de l'effondrement d'un système. La constitution et la perduration de ces friches dépassent alors le seul contexte de la ville concernée : ces friches signifient des pertes d'emplois et de richesse. La solution immédiate a concerné principalement la conservation de l'emploi et la préservation de la vocation industrielle des lieux en déshérence.

De fait, lorsqu'une inversion de la tendance à la fermeture des lieux de travail n'a pu être réalisée, ces friches industrielles sont souvent restées des espaces résiduels, une forme de verrues en » stand-by » (Andres et Bochet, 2007) sur les marges de la ville.

31 Ces friches créent alors des discontinuités dans le tissu urbain tout en contribuant à isoler davantage certains quartiers, en particulier les lieux d'habitat liés à l'activité initiale des friches. L'utilisation du terme derelict land dans le discours anglo-saxon est significative de cette image pessimiste.

Outre ces aspects, des éléments objectifs incitent les aménageurs du territoire et les planificateurs urbains à se détourner de ces friches avec des impacts sur leur 
environnement direct. Au niveau paysager, ces espaces à l'aspect répulsif se transforment parfois en lieux de prédilection pour des activités illicites. Dès lors, ces friches sont redoutées par les garants de la sécurité publique qui envisagent souvent une interdiction totale d'accès aux lieux. Ceci concourt encore davantage à leur isolement. Le maintien à l'abandon d'une friche peut accentuer la dévalorisation du quartier environnant, tant sur un plan social que physique, du fait des phénomènes de dégradation. Une conséquence directe peut être la perte de la valeur foncière de lîlot, voire des îlots environnants. Ceci s'inscrit dans un cercle vicieux avec le départ de nouvelles entreprises et un appauvrissement général liés à une perte de substance, économique, sociale, culturelle, civique (Rey, 2013).

\section{D'une logique d'espace craint à celle d'un enjeu pour le territoire}

33 Longtemps ignorées alors que la tendance était au développement, les friches urbaines sont cependant devenues des objets d'intérêt avec l'apparition, puis le renforcement du principe de développement durable: les villes doivent trouver le moyen d'être plus économes en termes de transports ou d'aménagement foncier. Ces espaces délaissés représentent un intérêt croissant pour satisfaire les besoins du renouvellement urbain (Masboungi, 1998).

34 Cependant, cette évolution s'est faite en intégrant plusieurs facteurs: le besoin fréquent de faire le deuil des activités initiales du terrain en friche et, de façon plus générale, la capacité à faire du recyclage urbain en inventant de nouvelles fonctions pour des bâtiments avec une architecture parfois marquée, voire à repenser le fonctionnement d'un quartier entier. Ces évolutions se sont réalisées en plusieurs temps: les premières reconversions de friches urbaines se sont souvent faites en réaction à un besoin identifié dans l'urgence, et s'intégraient alors dans une échelle relativement restreinte, correspondant au quartier. Ces réponses spontanées à un problème urbain n'ont pas toujours bénéficié de longévité et les bénéfices de la reconversion ont pu apparaître faibles alors que les prémices de la crise économique s'annonçaient. Ceci a pu contribuer, a contrario, à accentuer la déprise d'un territoire (Musika, 1994).

35 À l'inverse, lorsque la mise en perspective du projet de reconversion de la friche s'est inscrite dans un cadre plus large, les requalifications de sites ont connu leurs premiers succès pérennes (Chasseriau, 2004). Alors que la crise économique s'accompagnait de nombreux maux sociaux matérialisés dans la relation des citadins à la ville, avec une dégradation du bâti ou une ségrégation spatiale accrue, les tentatives de réponses se sont multipliées (Plaza, 1999). D'abord parcellaires, les solutions mises en place ont évolué pour s'intéresser à la ville dans son ensemble, en évaluant les besoins présents et futurs, et en interconnectant les problèmes identifiés pour y apporter une réponse qui se veut globale. L'avènement du projet urbain a transformé la façon d'appréhender la ville et de répondre à ses maux, ou à ses besoins, dans le cadre d'une concurrence accrue entre les villes.

Les friches urbaines ont alors bénéficié d'un regain d'intérêt. D'espaces subis à sécuriser, elles sont fréquemment devenues du foncier convoité. Les dernières friches urbaines industrielles ont ainsi pu être rapidement reconverties. Après des années d'attente, elles ont parfois permis à la commune concernée de bénéficier d'un changement d'image dépassant largement l'échelle locale. On a ainsi pu mettre en 
évidence un effet de levier qui a joué le rôle d'une locomotive pour sur le dynamisme territorial (Gravari-Barbas, 2004).

De fait, certains propriétaires de friches choisissent maintenant d'en faire un atout lié à la pénurie de foncier disponible. L'État français met ainsi sur le marché foncier une partie de ses biens dans le cadre de la Réforme Générale des Politiques Publiques (RGPP) : près de 2000 sites ont été mis en vente en huit ans pour rationaliser le patrimoine tout en renflouant les caisses publiques. Au début des années 2010, les principales administrations concernées étaient les ministères de la défense $(30 \%)$, du budget (26\%) et de l'éducation ( $22 \%$ ) (Journal Sud-ouest, édition électronique du 5 août 2010).

Bien que ses friches fassent l'objet d'une catégorisation spécifique, l'offre immobilière du ministère de la défense est aussi ancienne que celle des autres ministères et n'a pas toujours été réalisée dans le contexte de la RGPP (Carré, 2001). Parfois les contraintes géomorphologiques ont justement imposé un constant recyclage foncier car la ville ne pouvait plus s'étendre sur ses franges et les friches militaires restaient le seul foncier disponible pour accueillir de nouvelles fonctions. C'est notamment le cas de Grenoble (Grandvoinnet, 2011).

39 À l'instar des autres types de friches, les sites militaires en cessation d'activité n'ont pas été problématiques tant que leur fréquence d'apparition et leur nombre restaient faibles et quele cycle de renouvellement urbain permettait à la fois de compenser les effets du départ de l'Armée, et d'intégrer ces nouveaux espaces disponibles dans l'espace urbain.

40 Cependant, à partir des années 1980, le nouveau contexte géostratégique pousse l'État français à rationaliser ses effectifs militaires sur le territoire national et à supprimer ou à transférer leurs activités. Un nombre massif de casernes urbaines a alors été fermé.

41 L'apparition de ces friches militaires urbaines, fréquemment d'une superficie importante, pose l'immédiate question de son devenir. Elle s'inscrit aussi dans une logique de compensation des impacts du départ des militaires en termes économiques et sociaux.

Dans un contexte économique global de crise qui les fragilise, les villes peinent à intégrer ces nouveaux espaces à reconvertir. De plus, ces sites longtemps restés à l'écart du développement urbain suscitent une certaine méfiance car les friches militaires urbaines héritées de la Seconde Guerre mondiale ne sont pas fréquemment reconverties et sont sujettes à la délinquance ou à des dégradations (Masboungi et all, 2006).

43 C'est un paradoxe d'affirmer que les immeubles militaires témoignent tous d'une certaine longévité. Dès lors, ils ont pour la population une valeur historique, tant sur le plan architectural que mémorial, car ils font partie intégrante de la ville. Ceci implique un attachement social particulier. Les implantations militaires, bien qu'imposées par l'État et parfois subies pour les territoires, car considérées comme des entraves au développement urbain, restent donc des héritages.

La fermeture des sites militaires s'est accentuée à partir des années 1990 puis avec la mise en place de la RGPP. Pour les futures friches militaires urbaines, les délais de cessation d'activité sont parfois de moins d'un an. Dans ce nouveau contexte de cessation d'activité du site militaire, au-delà de la stricte emprise foncière, la friche 
véhicule toutes les conséquences économiques et sociales du départ des militaires. Ces éléments portent à considérer la friche militaire urbaine avec une défiance particulière.

\section{La friche militaire, une exception?}

Souvent méconnue du fait du secret entourant les activités de l'armée, la nature des friches militaires mérite d'être précisée car elles peuvent avoir des points communs avec d'autres friches, notamment tertiaires ou industrielles. De plus, ce sont parfois les seules ressources foncières encore disponibles au cœur de l'urbain alors que les autres types de friches peuvent être plus éloignées et donc plus contraignantes pour la ville compacte. Cependant, au-delà de ce constat, le patrimoine cédé par les Armées est très varié. Ainsi, entre 2000 et 2006, plus de 600 sites, dont 132 casernes, 13 bases aériennes, 12 hôpitaux, 3 bases de sous-marins, une centaine de forts ou de batteries et une base de missiles ont été mis en vente pour une superficie totale de 6000 hectares. Ce mouvement s'est accentué avec les Lois de Programmation Militaire (LPM) suivantes.

Du fait de l'importance du patrimoine immobilier mis sur le marché et du besoin de rééquilibrage du budget de l'État dans un contexte de RGPP, les sites sont parfois moins facilement négociables que des biens courants. Cependant, ceci ne constitue pas en soi une spécificité de la friche militaire, car toutes les friches publiques sont soumises à cette logique de vendre au meilleur prix pour l'État. De fait, les acquéreurs potentiels ne sont pas nombreux, bien que le ministère de la défense mette en œuvre, depuis de nombreuses années, une politique immobilière qui instaure des règles et procédures spécifiques pour améliorer les conditions de cession de ses biens.

\section{Un patrimoine divers avec des inconvénients propres}

Entre 1987 et 1997, 610 cessions de terrains militaires avaient déjà été réalisées pour un montant moyen de 250 millions de francs (Soit environ 38 millions d'Euros) par an. La plupart des régions d'implantation militaire traditionnelle avait alors connu des fermetures de sites et des reconversions foncières. Ces dix années de vente de terrains militaires ont fait l'objet d'une mise en perspective qui a permis une ébauche de classification (Guelton, 1998) qui reste aujourd'hui valable dans le cadre des LPM en cours : trois grands ensembles se distinguent en fonction de la facilité à reconvertir ces sites militaires (cf.figure 2):

48 Un premier grand ensemble correspond à des friches marquées par l'armée avec des bâtiments en ruines qui conservent des traces de la présence militaire et notamment des pollutions spécifiques ou une architecture marquée. Ces terrains, à l'écart des voies de circulation, présentent un intérêt moindre pour une reconversion urbaine.

Cet ensemble comprend des éléments remarquables tels des forts massifs, impossibles à reconvertir, sauf en sites touristiques entretenus par des associations de sauvegarde du patrimoine. C'est notamment le cas de la chaîne des forts dits « de Vauban » dans les Alpes. Les forts des littoraux méditerranéens, lorsqu'ils sont en friches, sont également contraignants à reconvertir car leur acquisition par une commune ou un particulier impliquerait l'applicabilité de la Loi littoral ${ }^{1}$ et une offre foncière associée moins intéressante. De nombreux Blockhaus du Mur de l'Atlantique restent également des verrues paysagères coûteuses à conserver mais difficilement reconvertibles. 

passées et qui conservent une forte valeur symbolique: le plateau d'Albion a été dépollué et sécurisé mais ne peut être totalement reconverti, car l'Armée veut conserver le secret des activités qui s'y sont déroulées.

De nombreux camps de tirs, terrains d'entraînement ou zones d'exercices complètent ce premier ensemble. Fréquemment éloignés des zones urbaines, ces sites en inactivité sont fréquemment rendus à l'état de nature, avec une prise en charge par l'office National des Forêts (ONF) une fois dépollués et/ ou sécurisés.

52 À l'inverse, des emprises abandonnées sont parfois longtemps ignorées avant d'être vendues: la base de sous-marins de Saint-Nazaire a été achetée par la municipalité pour l'équivalent de 122000 Euros dans le cadre du projet urbain dont l'ampleur a permis d'effacer l'image négative de cette base, construite par les Allemands, et qui fut la cause du bombardement de la cité et de sa destruction en 1945 (Prelorenzo, 2014). De fait, pendant la période de croissance et de développement urbain, jusqu'en 1975, la base sous-marine resta un stigmate de la seconde guerre mondiale et sa reconversion présentait peu d'intérêt. De friche militaire, elle se transforma en terrain vague industriel offrant des opportunités pour les populations marginalisées.

53 Avec le développement du projet urbain inscrit dans une logique d'ensemble, elle revêt un autre intérêt après cinquante ans d'abandon: la ville, sinistrée parla baisse d'activité des chantiers navals, cherche un nouveau souffle. En 1994, la municipalité initie le projet Ville-Port pour cette friche. Entre 1996 et 2000, la première phase des travaux permit d'y implanter des équipements liés au tourisme et à la culture ainsi que des logements avec l'ouverture et la remise en eau de de quatre alvéoles. En 2002, la réhabilitation de la base et du quartier voisin se poursuivit avec la transformation de nouvelles alvéoles et, à partir de 2005, de nouveaux lieux accueillirent des concerts et des expositions. La réalisation du projet s'est poursuivie jusqu'en 2017 pour valoriser et renforcer la vocation portuaire et maritime de l'avant-port en requalifiant son front de mer.

54 À l'exception d'une architecture marquée lorsque la base de Saint-Nazaire a commencé à être reconvertie, rien ne la distinguait d'une friche industrielle ou portuaire, car elle présentait les mêmes problématiques de pollution et d'occupation illicite.

55 Un deuxième grand ensemble comprend les friches militaires bâties tels des casernements, des logements ou des centres mobilisateurs. Ce ne sont pas des friches très marquées par l'Armée et qui présentent une certaine densité.

56 Ces bâtiments, souvent du XIXème siècle, initialement édifiés en lisière de ville, laquelle a progressivement disparu avec l'urbanisation, sont aujourd'hui en première couronne. La présence de ces implantations militaires crée de fortes discontinuités dans le tissu urbain, souvent des préfectures ou des sous-préfectures. En bon état et situés dans le centre ancien de grandes villes, ils trouvent rapidement preneurs aux prix du marché ; les mairies ou les établissements publics achètent ceux en mauvais état à des prix plus faibles. Ces immeubles représentent une bonne opportunité d'aménagement malgré la nécessité d'une importante réhabilitation.

57 Cependant, l'intérêt pour ces bâtiments est parfois incertain: à Amiens, le site de la caserne Dejean a été délaissé pendant vingt ans à l'issue de la dissolution du 8eme Régiment d'infanterie en 1993, car la ville ne voulait pas acheter cette caserne au prix proposé par l'État $(2,6 \mathrm{M} €)$. Un promoteur privé a finalement acquis l'emprise située à 
proximité de la gare. La finalisation des travaux a permis de mettre sur le marché immobilier de 89 logements de standing, 117 logements étudiants et 85 logements sociaux.

La superficie des actifs immobiliers du ministère de la défense parfois situés dans de petites communes est fréquemment le principal obstacle à une cession rapide car l'absence d'un projet de reconversion à l'échelle de l'emprise et le défaut de financements sont problématiques. Ce fut le cas de la caserne classique de La Fère, dans l'Aisne. Ce bâtiment remarquable, dit « des Prussiens », ne s'intégrait pas dans le cœur de la petite ville car trop grand par rapport à la taille du bourg: l'emprise des bâtiments militaires représentait les trois quarts de la surface de la ville. La commune, avec l'aide de l'État, a racheté progressivement les quatorze bâtiments, la Ville s'occupant de la rénovation, le département finançant l'achat et la voirie. Pour ouvrir le site militaire à la ville, il a fallu abattre les murs d'enceinte et raser des bâtiments, seuls les vestiges des fortifications Vauban ont été préservés. Quinze ans de travaux ont été nécessaires pour rénover le site aujourd'hui découpé en ateliers pour les artisans et les PME.

Si la Fère reste la ville la plus pauvre du département de l'Aisne ( 25 \% de chômage), elle a su reconstituer la cohérence architecturale de son bâti tout en créant 127 emplois. Elle n'a cependant pas retrouvé le niveau d'activités économiques antérieur à la reconversion du site. Ce n'est pas une spécificité liée au site militaire, la situation aurait été identique s'il avait fallu reconvertir une usine qui était le premier employeur de la ville.

Dans des villes plus importantes, ces immenses constructions restent des handicaps, en particulier lorsqu'elles sont classées, certains sites cumulant les inconvénients : à Bonifacio, le site de la caserne Montlaur, sur 3 hectares, comporte des bâtiments classés de la période génoise avec des caractéristiques architecturales intéressantes, mais la commune peine à envisager une opération appropriée car les projets de reconversion sont incohérents entre eux. La caserne est désaffectée depuis le début des années 1990, mais les premières esquisses de projet n'apparaissent que depuis 2012.

61 Les espaces destinés à la "vie sociale " des garnisons, cercles-mess ou hôpitaux militaires, moyennement densifiés et proches du centre urbain, se reconvertissent aisément dans la logique d'une friche tertiaire. Ainsi, l'ancien hôpital militaire Hyacinthe Vincent de Dijon est devenu un écoquartier avec 190 logements intramuros, de même l'ancien mess des officiers de Colmar, sur le site de la caserne Rapp, est reconverti en un pôle santé de $3200 \mathrm{~m}^{2}$.

Parfois, le départ de l'armée est un bienfait pour la ville car il lui rend en quelque sorte l'intégralité de son urbanité. Certaines villes en ont profité bien avant la structuration de la cession des friches militaires; Strasbourg, ville frontière, a connu une forte implantation militaire qui a progressivement cessé entre 1922 et 1958 . Ce foncier, cédé à la ville, a notamment permis de construire le quartier bourse et de disposer d'une centaine d'hectares pour des habitations, des commerces, des bureaux, l'université et plusieurs milliers de logements, du moyen au haut standing pour valoriser la ville qui accueillait depuis peu les institutions européennes. Ainsi, Strasbourg a bénéficié de l'urbanité de ses friches militaires pour affronter les défis urbains des années 1960, mais aussi pour affirmer son statut de métropole européenne et de capitale régionale.

Un dernier grand ensemble regroupe des emprises partiellement bâties avec des friches à l'orée de la ville. Ces grandes casernes, aérodromes ou centres de transmission offrent 
de réelles perspectives d'aménagement car localisés en zone urbaine constructible. Ces friches militaires sont assimilables à des friches quelconques à l'exception de contraintes de pollution spécifiques. Le cas de la base aéronavale de Fréjus est caractéristique : dans les années 1970, environ 1000 militaires et civils travaillaient sur ce site situé en bord de mer, dans le prolongement du port de plaisance et du centreville. En 1983, le ministre de la défense annonça une diminution de $30 \%$ des effectifs de la base avec le début de la procédure d'abandon du site qui prit fin le 30 juin 1995.

La municipalité, intéressée depuis longtemps par ce foncier pour des activités touristiques, se heurta cependant à la Loi littoral pour la réalisation de ses projets initiaux. Le site devint donc une "base nature » avec des infrastructures sportives et une plage sécurisée.

On trouve également dans cet ensemble des terrains nus, non viabilisés, qui sont de grands terrains d'exercice ou des parcelles en périphérie des villes. Ils peuvent être utilisés pour de nouvelles constructions ou des aménagements paysagers qui participent à la qualité de vie urbaine. Ainsi, Metz totalise à elle seule 3500 hectares de forêts militaires, en plus de ses autres friches urbaines. Ces terrains sont entretenus par l'ONF jusqu'à leur éventuelle cession.

Figure 2 : la typologie des friches militaires en fonction de leur aptitude à être reconverties

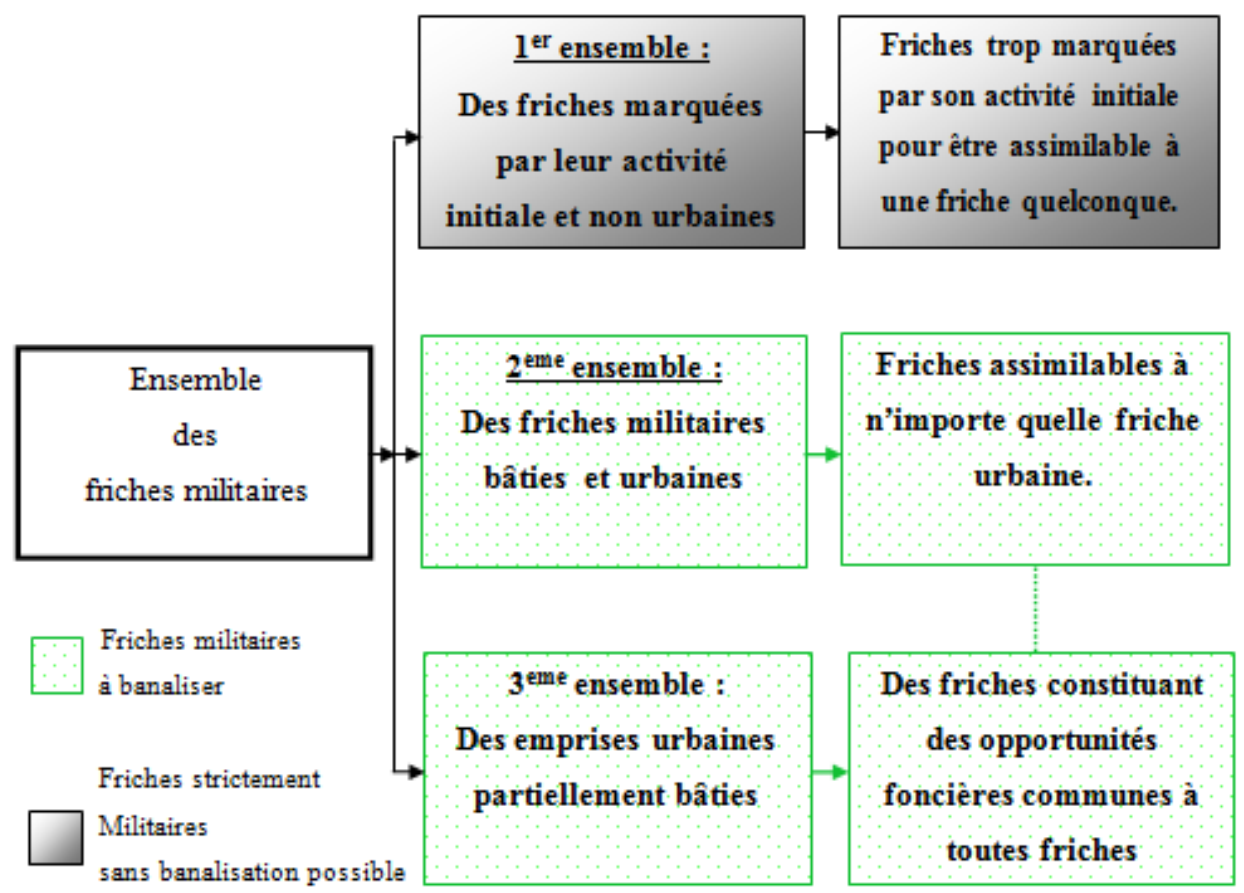

Source : Lotz-Coll, 2017

\section{La friche militaire, quelle place dans le projet urbain?}

Au milieu des années 1980, le ministère de la défense français possédait environ 250000 hectares, soit près d'un dixième du domaine public de l'État. Parmi ces emprises, 19000 hectares environ étaient situés en agglomération ou en première couronne. Ce sont ces sites qu'il faut s'efforcer d'intégrer à l'espace urbain. 


\section{Le départ des militaires, un traumatisme à compenser}

Entre 2009 et 2019, plus de 180 sites militaires doivent être cédés par le ministère des armées. En termes de marché de l'emploi local, ceci représente la suppression de 108 000postes civils ou militaires. Au-delà de l'enjeu de développement durable lié à l'avenir de ces sites, l'impact socio-économique du départ des militaires pose de véritables questions de (re)développement territorial. En effet, les LPM provoquent de sévères déséquilibres dans les bassins d'emplois : au-delà du strict emploi du militaire, beaucoup d'activités indirectes sont concernées par la présence des familles de militaires, les activités économiques externalisées par les armées, et toute une dimension d'aménagement du territoire avec une carte scolaire liée à celle militaire et parfois des transports.

Ainsi, pour Nancy, les mesures d'adaptation prévues par les Armées en 1997-1998 eurent un impact remarquable (Groupement ACT Consultants-FNAU services, 1997) pour les trois zones d'emploi de Nancy, Toul et Lunéville qui ont chacune perdu un régiment :

9 la population militaire totale avec familles est estimée à 23000 personnes, soit $4 \%$ de la population sur ces zones (INSEE 1990); pour la seule ville de Nancy, ceci représente 6300 personnes, soit $2 \%$ de la population de la zone d'emploi ;

La population scolarisée dans les trois zones d'emploi est de 6000 enfants (soit $50 \%$ des enfants du primaire ;

Pour la seule ville de Nancy, 1883 logements privés sont utilisés par des militaires, la vacance des logements sur Nancy étant déjà de $9 \%$ avant le départ des militaires ;

Les dépenses annuelles des trois régiments dissous dans les trois zones d'emplois atteignent $1080 \mathrm{MF}$, trois entreprises réalisant $10 \%$ de leur chiffre d'affaires annuel avec l'armée.

3 Ce départ est alors vécu comme une nouvelle menace de crise régionale : la LPM de 1997-2002 toucha durement la Lorraine où le poids de l'armée était important avec 40000 militaires dans 18 villes de garnison. C'est donc un double-traumatisme qui a également pu être observé sur la quasi-totalité du territoire français.

\section{La friche militaire urbaine, une opportunité ?}

74 Longues à reconvertir, les friches militaires des années 1980 ont souvent laissé une image négative. Le premier enjeu pour le ministère des armées est le pari opérationnel de rassurer et d'accompagner les acheteurs potentiels. Il s'agit d'occulter la qualification de «militaire» de la friche en insistant sur ses atouts urbains et en démystifiant les activités qui y ont eu lieu. Il a donc fallu adapter un outil immobilier diversifié intégrant les contraintes du secteur privé. À l'instar d'autres entreprises publiques grands propriétaires fonciers, le ministère des armées a su trouver une gestion immobilière appropriée. De fait, parmi les propriétaires fonciers de l'État, le ministère bénéficie de mesures dérogatoires. Ainsi, les textes réglementaires lui laissent la possibilité d'initier les procédures d'aliénation et une structure a été créée dans ce but en 1987, la Mission pour la Réalisation des Actifs Immobiliers(MRAI), qui mène, en concertation avec France domaine, les procédures de négociation des 
immeubles en vente. Son principal rôle est la négociation de la vente des biens immobiliers devenus inutiles aux armées avec une intervention dans trois domaines : es collectivités locales n'ont pas pu intégrer la future friche dans un projet urbain global. La MRAI prend alors le relais en engageant un processus dont la finalité est d'éviter la vente à l'euro symbolique. Pour faire émerger un projet de reconversion durant le temps de l'avant-friche, le dossier est souvent confié à un urbaniste qui travaille avec un comité de pilotage ad hoc constitué du préfet, de représentants de la commune, de la région et du département, des principaux acteurs intéressés et des administrations. Indépendamment des contraintes architecturales, la situation des friches militaires leur confère souvent une valeur territoriale les cités se sont construites autour d'elles, selon les usages et les représentations en vigueur et la caserne est fréquemment la première "entreprise" locale et, en dépit d'un fonctionnement autonome à l'écart de la communauté urbaine, une symbiose humaine et économique existe. La reconversion des terrains militaires permet alors un travail remarquable entre les administrations et le secteur privé : l'urgence crée des intérêts communs, la recherche de projets consensuels et l'adaptation des méthodes d'interface. La vocation publique des emprises succède souvent à leur usage militaire, la continuité de l'usage collectif donnant en quelque sorte une image institutionnelle au nouvel occupant.

Certains maires se sont empressés de saisir l'opportunité offerte: Brest métropole a bénéficié de la libération de terrains de l'armée sur le plateau des Capucins pour créer sur 16 hectares un nouveau quartier inscrit dans une dynamique de développement durable et de renouveau urbain. L'aménagement de ce plateau permet de valoriser les fonctions métropolitaines autour de la recherche marine, de l'économie, de la culture, et de l'habitat : $39000 \mathrm{~m}^{2}$ sont consacrés aux activités tertiaires et $41000 \mathrm{~m}^{2}$ à l'habitat, avec plus de 500 logements. L'ensemble représentera, à terme, plus de 1000 emplois avec $40 \%$ d'activités tertiaires et de commerce, $20 \%$ d'équipements publics et culturels et le reste en habitat.

Les premières reconversions de sites militaires étaient trop marginales pour qu'un programme d'ampleur soit mis en place. Cependant, des aides ont pu être apportées au niveau national, mais sans s'inscrire systématiquement dans une logique d'aide aux sites militaires en reconversion. Pour les reconversions les plus récentes, l'État a mis en place un dispositif permettant aux communes les plus touchées d'envisager plus sereinement la reconversion de leurs sites militaires en bénéficiant à la fois d'études et de financements. Ceci s'inscrit dans une logique de compensation du départ des militaires. 


\section{L'effet levier de la reconversion des friches militaires}

81 Des études (Groupement ACT Consultants-FNAU services, 1997) montrent que l'impact d'une reconversion de friche militaire sur l'attractivité d'un territoire se mesure grâce aux retombées de l'opération de requalification en termes d'image, de développement économique et social, ou encore de dynamisme démographique. Ainsi, la création de zones d'aménagement concertées (ZAC) permet de réutiliser des sites militaires pour l'intérêt général. Le quartier de La Part Dieu à Lyon et Compans Cafarelli à Toulouse sont exemplaires et leur passé militaire est aujourd'hui oublié.

Les projets de reconversion peuvent donc être des leviers puissants d'attractivité s'ils répondent aux besoins du territoire et à l'enjeu urbanistique de renouvellement urbain : la reconversion positionne un territoire, en particulier dans un contexte de concurrence interurbaine accrue, et pourra contribuer à diminuer les conséquences du départ de l'armée.

De fait, les opportunités économiques offertes par la reconversion des friches militaires sont nombreuses: création de logements et arrivée de nouvelles populations, développement de nouvelles filières, consolidation d'un secteur d'activité dit historique, implantation d'équipements structurants ou tourisme d'affaires intéressé par le site reconverti, etc. La reconversion des friches militaires témoigne d'une remarquable mixité fonctionnelle (Lotz, 2017). Au-delà de son urbanité, la friche militaire urbaine offre donc un éventail varié de possibilités de reconversion et propose de nombreuses perspectives pour la réalisation d'un effet levier. Ceci concourt par ailleurs à sa réintégration dans la trame urbaine.

Pour parvenir à cet objectif, des conditions favorables sont nécessaires. Ainsi, alors que les évolutions urbaines récentes ont confirmé que la reconversion d'une friche n'est pas acquise a priori pour un terrain militaire, elle doit s'envisager dans le cadre d'un projet d'ensemble pour la ville, en particulier lorsqu'il s'agit de friches d'ampleur. La démarche de projet urbain propose un cadre adéquat pour réaliser une synergie entre la trame urbaine déjà existante et les nouvelles fonctions de la friche reconvertie (cf. figure 3). On peut alors parler de projet urbain de reconversion d'un site militaire, notion qui donne à la requalification tout son poids dans le cadre de la trame urbaine globale.

Au-delà des projets de requalification planifiés, que ce soit pour des opérations ou pour le site militaire-lui-même, les notions de réversibilité des projets déjà initiés et de complémentarité avec les opérations en cours restent cependant déterminantes. 
Figure 3 : le potentiel de la friche dans le cadre du projet urbain.

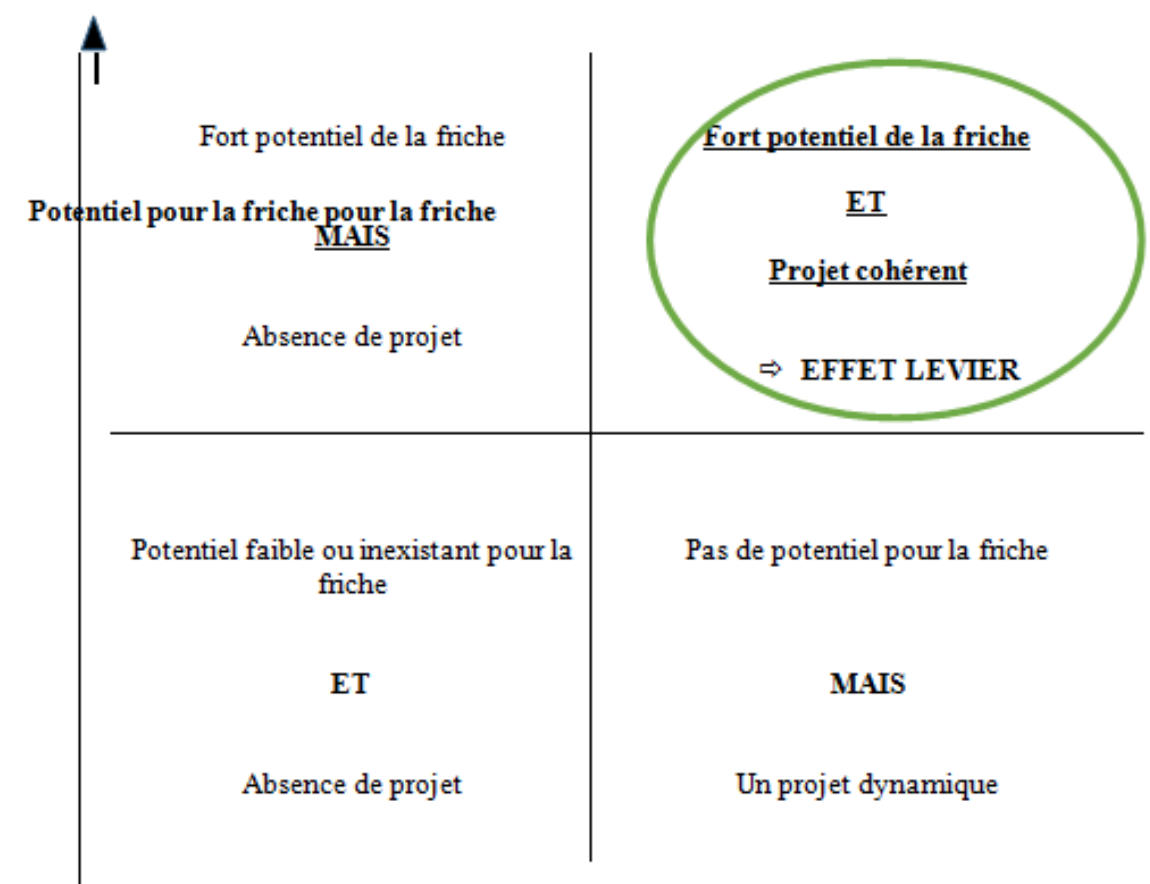

Projet pour la friche

Source : Lotz-Coll, 2017

Néanmoins, si le projet urbain est un processus qui permet aux friches militaires d'être reconverties, d'autres facteurs interviennent dans le succès de la reconversion (ibid.). Ils s'envisagent par une déclinaison plus fine du projet urbain et en intégrant des points spécifiques assimilables à des éléments de contexte. Ainsi, associée à la volonté de requalifier la friche militaire, l'existence d'une structure porteuse du projet de reconversion à l'échelle du territoire détermine le succès de l'opération. Ce sont souvent les communautés de communes ou les Sociétés d'Economie Mixte (SEM) locales qui permettent un meilleur portage financier et technique du projet. Elles vont aussi pouvoir favoriser l'installation d'acteurs institutionnels et de vecteurs de stabilité dès la fin de la reconversion (ibid.) : l'investissement financier réalisé pour la rénovation des infrastructures d'accueil pourra être compensé par l'attraction de nouvelles entreprises désireuses de bénéficier de ce contexte de confiance dans l'avenir de la reconversion. Produits d'appel et vecteurs d'image complètent alors le succès de la reconversion du site militaire en détournant l'attention de la fonction initiale du site et en le distinguant dans un cadre concurrentiel. Ainsi Noyon, dans l'Oise, a transformé en 2011 sa friche militaire de 47 ha en un campus économique novateur, Inovia.

Dans le cadre des diagnostics indispensables à la définition des objectifs de la reconversion et à la mise en place du projet de requalification de la friche militaire, il importe également de prioriser les actions à entreprendre en hiérarchisant les objectifs à atteindre, tels le maintien des habitants ou la création d'emplois : ainsi, à Noyon, la reconversion du centre équestre a été abandonnée pour consacrer les crédits associés à un équipement commercial (ibid.).

De même, lorsque les diagnostics ont été réalisés lors de la définition du projet de reconversion, il importe de les intégrer stricto sensu pour garantir les résultats de 
l'opération. À l'inverse, l'intégration forcée de réponses à des besoins non identifiés peut concourir à un échec comme ce fut le cas pour les locaux commerciaux de la caserne Lefebvre, à Mulhouse, dont le besoin n'était pas avéré et qui sont restés vides à l'issue de la reconversion (ibid.). L'image d'ensemble du succès de la reconversion peut alors être remise en question.

Outre ces éléments génériques applicables à toutes reconversions de friche, d'autres points particuliers concernent strictement les friches militaires dans un contexte global de crise économique et sociale : bien que le contexte financier de la reconversion soit souvent contraignant, il importe de laisser au projet urbain de reconversion d'une friche militaire une souplesse qui lui permette de s'adapter aux réalités du territoire vécu. Ainsi Joigny, dans l'Yonne avait davantage besoin d'une pépinière d'entreprises que d'un musée. Au-delà du financement courant de la reconversion de friches, il s'agit aussi de disposer de ressources financières permettant d'intégrer les besoins de rénovation spécifiques des sites des armées telle la mise aux normes des réseaux domestiques qui obéissaient jusqu'alors aux règles militaires.

De fait, la reconversion a aussi des impacts en matière d'image et de renommée ; ainsi, le marketing des grandes villes se fait sur la base de projets urbains ambitieux et la reconquête de la friche redynamise le territoire tout en revalorisant son image auprès des investisseurs et des populations. La ville de Montpellier a ainsi pu renforcer sa centralité avec son Polygone (centre commercial, bibliothèque-ludothèque et logements), espace de 11 onze hectares achetés à l'Armée en 1972 (Dubois-Maury, 1985). La ville poursuit cette logique avec la reconversion de l'École d'Application de l'Infanterie, projet fortement médiatisé.

91 De même, Lorient ancien grand port militaire, a reconverti la base de Kéroman, plus grande base de sous-marins d'Européen un pôle de course au large et en une destination touristique et de loisirs qui est devenue une destination incontournable $\mathrm{du}$ territoire.

92 L'effet levier de la reconversion peut alors se décliner par le biais de trois grands items, l'attractivité résidentielle, l'attractivité économique et l'effet d'image de la ville menant le projet de reconversion. Ces trois effets sont fréquemment interconnectés. De plus, dans le cadre de l'appréciation de cet effet levier, un élément remarquable a également été mis en évidence (Lotz, 2017.) : il s'agit pas de compenser nombre pour nombre les emplois perdus avec le départ des militaires car l'effet levier réside davantage dans le fait d'initier une dynamique d'ensemble qui profitera à moyen, voire long terme, à la ville et à son territoire.

Dans le cas particulier des petites villes qui choisissent de reconvertir leur site militaire, il s'agit par ailleurs d'une opération qui dépasse largement la stricte reconversion d'un site et qui nécessite un travail d'ensemble sur l'urbain, dont les résultats ne peuvent s'envisager à la simple lecture de critères objectifs. La reconversion du quartier Abescat, caserne militaire de Joigny, est caractéristique avec des actions qui ne se limitent pas au seul site militaire tel l'aménagement touristique des berges (ibid.).

94 Au même titre que la reconversion d'autres types de friches, la reconversion d'une friche militaire peut donc s'accompagner d'un véritable effet levier, d'autant plus manifeste qu'il permettra souvent d'initier un cercle vertueux et de (re)donner de l'optimisme dans des territoires parfois doublement sinistrés. 


\section{Conclusion}

\section{BIBLIOGRAPHIE}

AMBROSINO C., ANDRES L. (2008), « Friches en ville : du temps de veille aux politiques de l'espace. », Espaces et sociétés, 2008/134, pp. 37-51.

URL :www.cairn.info/revue-espaces-et-societes-2008-3-page-37.htmlconsulté le 7 mars 2018.

ANDRES L., AMBROSINO C. (2008), « Régénération culturelle et mutabilité urbaine : un regard franco - britannique », in Leriche F., Daviet S., Sibertin- Blanc M. et Zuliani J.M. (dir.), L'économie culturelle et ses territoires, Toulouse, Presses Universitaires du Mirail, 250p.

ANDRES L.(2010), « Reconquête culturo-économique des territoires délaissés : de l'importance du temps de veille et de ses acteurs transitoires », Méditerranée, $2010: 114,012$

URL : http://journals.openedition.org/mediterranee/4342consulté le 11 mai 2018.

BAIROCH P. (1985), De Jéricho à Mexico. Villes et économie dans l'histoire, Paris, Gallimard, 714p. BENEVOLO L. (1993), La Ville dans l'histoire européenne, (préface de J. Le Goff), Paris, Le Seuil, 284p.

Carnets de géographes, 11 | 2018 
BIANCHETTI D.(1985), « Aree industriali Dismesse : primipercorsi di ricerca/friches industrielles : premières voies de recherche », Urbanistica. (ITA), 1985/81, pp 83-99.

CHASSERIAU A. (2004),» Au cœur du renouvellement urbain nantais : la Loire en projet », Norois, $192 / 2004 / 3$

URL : http://norois.revues.org/899 consulté le 30 septembre 2016.

COMMUNAUTES URBAINES DE FRANCE (2010), Les friches, au cœur du renouveau urbain : les communautés urbaines face aux friches : état des lieux et cadre pour agir, 211p.

DAVIET, S.(2006), « L'évolution du concept de reconversion : de la substitution d'activité au redéveloppement des territoires ", in Daumalin, X. (dir.), Daviet, S. (dir.) et Mioche, P. (dir.), Territoires européens du charbon : des origines aux reconversions. Paris, Publications de l'Université de Provence, pp. 243-255.

DORIER-APPRILL É. (dir.) (2001), Vocabulaire de la ville. Notions et références, Paris, éditions du Temps, 191p.

DUBOIS-MAURY J. (1985), « Stratégies foncières des forces armées. Les échanges compensés »,Études foncières, 1985/26, pp. 13-17.

FERNANDEZ X. ET CORNES P. (1990), Barmi, une ville méditerranéenne à travers l'histoire, Rennes, éditions Ouest-France, collection Villes du monde à travers l'histoire, 70p.

GROUPEMENT ACT CONSULTANTS-FNAU SERVICES (1997),La reconversion urbaine des sites militaires : contexte, enjeux, et propositions d'aide à la décision et à l'action : rapport final, 34p.

GINET P.(2011), « Restructurations d'espaces militaires et développement des territoires », Revue Géographique de l'Est, 2011/vol. 51/1-2

URL :http://rge.revues.org/3219 consulté le 7 avril 2017

GIRAUD H. (2006), « Les friches ferroviaires très convoitées », Les Échos,31/03/2006

URL :www.lesechos.fr/31/03/2006/LesEchos/19638-148-ECH_les-friches-ferroviaires-tresconvoitees.htm\#szhiY4QzxeVg5vTt.99 consulté le 8 janvier 2015

GODET O. (2007), Patrimoine reconverti. Du militaire au civil, Paris, Scala et Ministère de la Défense, 271p.

GRAVARI-BARBAS M. (1998),» le festival market place » ou le tourisme sur le front d'eau. Un modèle urbain américain à exporter », Norois, 1998/29, pp. 261-278.

URL :http://www.persee.fr/doc/noroi_0029-182x_1998_num_178_1_6868 consulté le 18 novembre 2016

GRAVARI-BARBAS M. et JACQUOT S. (2007), «L'événement, outil de légitimation de projets urbains : l'instrumentalisation des espaces et des temporalités événementiels à Lille et Gênes ", Géocarrefour, 2007, vol. 82/3,

URL :http://geocarrefour.revues.org/2217 consulté le 19 août 2014

GRANDVOINNET P. (2011), « Les emprises militaires dans l'urbanisme grenoblois du XXe siècle : des opportunités foncières au patrimoine paysager », In Situ, 2011/16

URL : http://journals.openedition.org/insitu/217 ; consulté le 11 juin 2018

GUELTON S. (1998), « Dix années de vente des terrains militaires », Études foncières,1998/79, pp. 19-20. 
JANIN C., ANDRES L. (2008), « Les friches : espaces en marge ou marges de manœuvre pour l'aménagement des territoires? », Annales de géographie, 2008, vol 5 n 663, pp. 62-81.

URL :www.cairn.info/revue-annales-de-geographie-2008-5-page-62.htm consulté le 25 septembre 2014

LACAZE J-P. (2006), La transformation des villes et les politiques urbaines : 1945-2005, Paris, Presse de l'ENPC, 255p.

HASCHAR-NOE N. « Action publique et reconversion durable d'une friche industrielle. L'exemple de Cap Découverte » : BESSY O. (dir.), Sport, loisirs, tourisme et développement durable des territoires, Voiron : Presses universitaires du sport, 2008, pp. 109-122.

LACOUR C. (1985), « La réinsertion urbaine des friches industrielles : la ville redécouverte », Revue d'économie régionale et urbaine, 1987/5, pp. 14-16.

LOTZ S. (2017), « l'effet levier de la reconversion des sites militaires dans le cadre du renouvellement urbain en France ", thèse de doctorat en urbanisme et aménagement du territoire réalisée sous la direction de Sonia Guelton, Lab'urba, Ecole d'Urbanisme de Paris, université Paris 12, 660 pages, 2 tomes

MASBOUNGI A. (1998) « Recycler le territoire », Projet Urbain,1998/15, pp. 3-5.

MASBOUNGI, A., GRAVELAINE (De) F., ISHERWOOD B. (2006), Birmingham: Faire la ville en partenariat: City renewal through partnership. Paris, éditions de la Villette, 159p.

MUSIKA S. (1994), «Évaluation des friches industrielles », Évaluer un terrain, 1994, p. 147.

NOURY A. (2004), «Le réaménagement des friches urbaines », Droit et politiques de renouvellement urbain, La documentation française, coll. Les cahiers du GRIDAUH, pp. 169-190.

PASCAL É., KOSTRZEWA J. (2017), « Patrimoine de la santé : vers une méthode de reconversion pour des sites historiques d'envergure urbaine », In Situ, 2017/31

URL : http://journals.openedition.org/insitu/14469 consulté le 17 février 2017

PLAZA, B. (1999), The Guggenheim-Bilbao Museum Effect: A reply to Maria V. Gomez: Reflective images: The Case of urban regeneration in Glasgow and Bilbao, International Journal ofUrban and Regional Research, 2008/23(3), pp. 589-592.

PRELORENZO C. (2011), « Patrimonialiser les bases de sous-marins et le Mur de l'Atlantique », In Situ2011/16,

URL :http://insitu.revues.org/312 consulté le 20 juin 2015

REY E.(2013), Régénération des friches urbaines et développement durable : vers une évaluation intégrée à la dynamique du projet, Louvain-la-Neuve, Presses universitaires de Louvain, 293p.

REY-LEFEBVRE I. (2015), « Le service public exploite sa manne immobilière », Le Monde, 23.02.2015

URL : http://www.lemonde.fr/economie/article/2015/02/23/le-service-public-exploite-samanne-immobiliere_4581583_3234.html\#M0qkAgPeAPKuGRRM.99 consulté le 20 juin 2015

SAFFACHE P.(2005), Glossaire de l'aménagement et du développement local, Paris, Éditions Ibis Rouge, 205p.

TIANO C. (2010), « Neptune : le discours de la méthode, La requalification de friches industrialoportuaires », Les Annales de la recherche urbaine,2010/106, pp. 63-73. 


\section{NOTES}

1. La loi relative à l'aménagement, la protection et la mise en valeur du littoral dite Loi littoral de 1986 encadre l'aménagement des côtes pour les protéger des excès de la spéculation immobilière. Les sites militaires, du fait de leurs activités spécifiques, ne sont pas soumis aux contraintes de cette loi qui s'applique dès la cessation de l'activité militaire.

\section{RÉSUMÉS}

Dans un contexte de réforme générale des politiques publiques et d'adaptation de l'outil de Défense aux nouvelles contraintes géostratégiques, les friches militaires sont de plus en plus nombreuses. Alors que la ville, autrefois gourmande d'espace foncier sur ses franges, s'efforce aujourd'hui de rationnaliser la trame urbaine dans une logique de développement durable, elle s'intéresse davantage à ses espaces délaissés et tente de mettre en place une logique de reconversion qui puisse s'appliquer à toutes ces friches, quelle que soit la nature de leurs activités initiales. Parmi ces sites en attente de reconversion, les fiches militaires urbaines pourraient offrir de réelles opportunités en étant requalifiées et ainsi participer au renouvellement urbain. L'article suivant propose d'envisager dans quelle mesure la friche militaire urbaine peut être une opportunité pour un territoire.

In a context of general reform of public policies and of adaptation of the tool of Defense to new geostrategical pressures, military brownfields are becoming more numerous. In the past, the city used easily the land space on its fringes. While it is today trying to rationalize the urban network in the frame of sustainable development. It is more interested in its derelict lands and it tries to set up a logic of urban regeneration which could be applied to all these wastelands, whatever is the nature of their initial activities. Among these sites waiting for reconversion, the urban military wastelands could give real opportunities as part of regeneration by being renewed and so participate to the urban renewal. The article below proposes to consider how the military urban brownfields can an opportunity for the territory.

\section{INDEX}

Thèmes : Carnets de recherches

Keywords : Wasteland, Leverage Effect, Urban Renewal, Territory's Attractivity

Mots-clés : friche, effet levier, renouvellement urbain, attractivité du territoire

\section{AUTEUR}

\section{STÉPHANIE LOTZ-COLL}

Docteure en urbanisme et aménagement du territoire

Chercheur associé, Lab'Urba

Ecole d'urbanisme de Paris - Université Paris - Est

stephanie.lotz.urba@gmail.com 